Federal Reserve Bank of Minneapolis

Research Department Staff Report 151

January 1992

\title{
ON THE EXISTENCE AND UNIQUENESS OF NONOPTIMAL EQUILIBRIA IN DYNAMIC STOCHASTIC ECONOMIES
}

\author{
Jeremy Greenwood \\ Federal Reserve Bank of Minneapolis \\ and University of Western Ontario
}

\author{
Gregory W. Huffman \\ University of California at Riverside \\ and University of Western Ontario
}

\begin{abstract}
The question of the existence and uniqueness of a stationary equilibrium for distorted versions of the standard neoclassical growth model is addressed in this paper. The conditions presented guaranteeing the existence and uniqueness of nontrivial equilibrium for the class of economies under study are simple and intuitively appealing, while the existence and uniqueness proof developed is elementary. Examples are presented illustrating that economies with distortional taxation, endogenous growth with externalities, and monopolistic competition can all fit into the framework developed.
\end{abstract}

The views expressed herein are those of the authors and not necessarily those of the Federal Reserve Bank of Minneapolis or the Federal Reserve System. 


\section{INTRODUCTION}

A great deal of work has recently been devoted to the study of economic environments which give rise to nonoptimal equilibria [see, for example, Coleman (1991), Greenwood and Huffman (1991)]. The analysis of these environments is not a straightforward application of results known from dynamic competitive analysis, as in Stokey and Lucas with Prescott (1989), since the resulting equilibria are not optimal. The usual problem in these environments is that individual agents fail to take into account how their actions influence the behavior of other agents. In order to solve an individual agent's dynamic program, one needs to know the equilibrium law of motion governing the aggregate state of the world, but to know this in turn requires knowledge of the outcome of decision-making at the individual level. Proving existence of equilibria can then be problematic, since in these representative agent models it can be difficult to establish that the individual and aggregate laws of motion for decision variables coincide along an equilibrium path. Various assumptions have been suggested to guarantee existence or uniqueness of an equilibrium. It is shown below that a relatively minor restriction on the nature of the technology, with no unusually restrictive assumptions placed on preferences, is sufficient to guarantee the existence and uniqueness of an equilibrium.

The environment studied below is very similar to the standard neo-classical growth model, with the notable exception that aggregate decisions can affect the decisions or resources available to each agent. Many dynamic stochastic tax experiments can be cast in this framework, especially the case where the resulting tax revenue is rebated back to agents in a lump-sum manner [see Greenwood and Huffman (1991)]. In addition, if there is a positive externality in the production technology whereby one agent's investment decisions can influence the productivity of other agents, this too can be cast in the framework described below.

The analysis conducted below borrows ingredients from Coleman (1991) and Greenwood and Huffman (1991). In particular, following the innovative work of Coleman (1991), the existence of equilibrium is established by constructing a monotone operator which maps the aggregate law of motion into itself. Coleman's (1991) strategy for proving existence and uniqueness is generalized and significantly simplified in three ways: first, by adopting a less restrictive and more intuitive set of assumptions on tastes and technology outlined in Greenwood and Huffman (1991); second, by employing a simpler line of argument to prove that the fixed point to the monotone 
operator is an equilibrium for the economy under study; and third, through the use of the elementary uniqueness proof developed here. The end result of combining components from the above two analyses is an existence and uniqueness proof that is much simpler than that contained in either work, despite the greater generality of the argument being pursued. Another benefit of the present approach is that the assumptions employed below would appear to be readily verifiable for a variety of environments.

\section{THE ECONOMIC ENVIRONMENT}

The economy under consideration is one which is very similar to the discrete-time standard neo-classical growth model. There is a continuum (of measure one) of identical agents who have preferences described as

$$
E\left[\sum_{t=0}^{\infty} \beta^{t} U\left(c_{t}\right)\right], \quad \beta \in(0,1),
$$

where $E[\cdot]$ is the expectation operator, $\beta$ is the discount factor, and $c_{t}$ represents consumption in period t. The momentary utility function $U(\cdot): \Re_{+} \rightarrow \Re_{+}$is strictly increasing, strictly concave, and twice differentiable and $U^{\prime}(0)=\infty$.

Each agent is endowed with the same production technology which is written in the following form:

$$
y_{t}=F\left(k_{t}, K_{t}, \eta_{t}\right)
$$

Here, $y_{t}$ represents output of the consumption good in period $t$, and $k_{t}$ is the agent's private input of capital into the production process which was chosen in period $t-1$. The variable $\eta_{t}$ is a random technology shock which is known at the beginning of period t. It is drawn from the bounded set $\Lambda$ and has a Markov distribution function which will be denoted as $G\left(\eta_{t+1} \mid \eta_{t}\right)$. The variable $K_{t}$ represents the average or per capita quantity of capital supplied by all agents in the economy. Each agent, being of measure zero, behaves as though his choice of capital stock $k_{t}$ has no influence on the average capital stock $\mathrm{K}_{\mathrm{t}}$. As is conventional, it is assumed that the production technology is increasing and concave in its first argument, and twice differentiable in its first two arguments. Other than the restrictions described below, no further constraints are placed on the technology with 
respect to the influence of the remaining two arguments.

Assumptions: $\forall \eta \in \Lambda$ :

$$
\mathrm{F}(0, \mathrm{~K}, \eta) \geq 0, \mathrm{~F}_{1}(0, \mathrm{~K}, \eta)=\infty, \forall \mathrm{K}>0 \text {, and } \lim _{\mathrm{K} \rightarrow 0} \mathrm{~F}_{1}(\mathrm{~K}, \mathrm{~K}, \eta)=\infty \text {. }
$$

$\exists \overline{\mathrm{K}} \ni \mathrm{F}(\overline{\mathrm{K}}, \overline{\mathrm{K}}, \eta) \leq \overline{\mathrm{K}}$.

For all $\mathrm{k}, \mathrm{K} \in[0, \overline{\mathrm{K}}]$,

$\mathrm{F}_{1}(\mathrm{k}, \mathrm{K}, \eta)+\mathrm{F}_{2}(\mathrm{k}, \mathrm{K}, \eta) \geq 0$,

and

$$
\mathrm{F}_{11}(\mathrm{k}, \mathrm{K}, \eta)+\mathrm{F}_{21}(\mathrm{k}, \mathrm{K}, \eta)<0
$$

These assumptions seem relatively innocuous. The first assumption places relatively standard restrictions on the production technology. The second merely places an upper bound on the level of output, or capital stock in the economy, which is satisfied for almost all parameterized versions of the concave technology. The third assumption places benign restrictions on how aggregate capital can affect the individual technology. It requires that the social marginal product of capital (or the sum of the marginal products of the individual and aggregate stocks) be positive. Additionally, it is necessary that the social returns to capital are diminishing in the individual capital stock. These properties need only hold locally "along an equilibrium path" where $\mathrm{k}=\mathrm{K}$. It is interesting to note that in a nonstochastic environment, Assumption (iii) would also be sufficient to guarantee a unique steady-state equilibrium. It would seem easy to check that these simple properties hold for a wide range of economic problems. Additionally, it should be recognized that these appear to be much less restrictive assumptions than those imposed on the production and utility functions in Coleman (1991). ${ }^{1}$

Finally, output in each period must be used for either consumption or investment purposes. Thus,

$$
c_{t}+k_{t+1} \leq y_{t}
$$

where $k_{t+1}$ is the amount of the consumption good used for investment at date $t$ which becomes productive capital in the following period. 


\section{EXISTENCE AND UNIQUENESS OF EQUILIBRIA}

The representative agent's dynamic programming problem for the environment under study can be cast as

$$
V(k, K, \eta)=\max _{k}\left\{U\left(F(k, K, \eta)-k^{\prime}\right)+\beta \int V\left(k^{\prime}, K^{\prime}, \eta\right) d G\left(\eta^{\prime} \mid \eta\right)\right\}
$$

where the aggregate $K$ evolves exogenously according to the nontrivial law of motion $K^{\prime}=Q(K, \eta)$. Let the optimal decision-rule associated with the above problem be denoted by $k^{\prime}=q(k, K, \eta)$. Standard arguments can be used to show that the unique interior solution for this decision-rule must satisfy the Euler equation (1) shown below.

$$
U^{\prime}\left(F(k, K, \eta)-k^{\prime}\right)=\beta \int U^{\prime}\left(F\left(k^{\prime}, K^{\prime}, \eta^{\prime}\right)-k^{\prime \prime}\right) F_{1}\left(k^{\prime}, K^{\prime}, \eta^{\prime}\right) d G\left(\eta^{\prime} \mid \eta\right)
$$

Now, the economy under study is one where all agents are identical. In equilibrium, therefore, the decision-rule regulating the individual's capital accumulation must coincide with the law of motion governing the evolution of the aggregate capital stock. The following definition makes this notion more precise:

Definition: A stationary equilibrium for the environment described above is a pair of functions $\mathrm{k}^{\prime}=\mathrm{q}(\mathrm{k}, \mathrm{K}, \eta)$ and $\mathrm{K}^{\prime}=\mathrm{Q}(\mathrm{k}, \eta)$ such that

(i) the decision-rule for each agent, $k^{\prime}=q(k, K, \eta)$, solves the optimization problem (P1), and

(ii) the individual's decision-rule is consistent with the law of motion for the aggregate per capita capital stock, or $\mathrm{q}(\mathrm{K}, \mathrm{K}, \eta)=\mathrm{Q}(\mathrm{K}, \eta)$.

From the above definition of an equilibrium, it is clear that if the aggregate law of motion for capital, $K^{\prime}=Q(K, \eta)$, exists then it must satisfy equation (2)

$$
U^{\prime}\left(F(K, K, \eta)-K^{\prime}\right)=\beta \int U^{\prime}\left(F\left(K^{\prime}, K^{\prime}, \eta^{\prime}\right)-K^{\prime \prime}\right) F_{1}\left(K^{\prime}, K^{\prime}, \eta^{\prime}\right) d G\left(\eta^{\prime} \mid \eta\right)
$$

where $K^{\prime \prime}=Q\left(Q(K, \eta), \eta^{\prime}\right)$ 
Proposition: There exists a unique stationary nontrivial equilibrium for the economy described above.

\section{Proof:}

\section{A. Construction of the Aggregate Law of Motion}

Consider the sequence of aggregate laws of motion $\left\{\mathrm{H}^{\mathrm{j}}(\mathrm{K}, \eta)\right\}^{\infty}{ }_{\mathrm{j}=0}$ that are generated recursively as follows: First define $\mathrm{H}^{0}(\mathrm{~K}, \eta) \equiv 0$. Second, define $\mathrm{H}^{\mathrm{j}+1}(\mathrm{~K}, \eta)$ to be the solution for $x$ in the Euler equation shown below

$$
U^{\prime}(F(K, K, \eta)-x)=\beta \int U^{\prime}\left(F\left(x, x, \eta^{\prime}\right)-H^{j}\left(x, \eta^{\prime}\right)\right) F_{1}\left(x, x, \eta^{\prime}\right) d G\left(\eta^{\prime} \mid \eta\right) .
$$

Thus, equation (3) implicitly defines an operator mapping the function $\mathrm{H}^{\mathrm{j}}$ into $\mathrm{H}^{\mathrm{j}+1}$. 2 Now assume that

$$
0 \leq \frac{\partial H^{j}(K, \eta)}{\partial K} \leq\left[F_{1}(K, K, \eta)+F_{2}(K, K, \eta)\right]
$$

which certainly holds for $\mathrm{j}=0$. The left-hand side of equation (3) is strictly increasing in $\mathrm{x}$, and the right-hand side is decreasing in $x$ by Assumption (iii) and equation (4). Hence, there exists a unique solution to equation (3). Note also that if the capital stock $\mathrm{K}$ increases by a unit, then output increases by the amount $\left[F_{1}(K, K, \eta)+F_{2}(K, K, \eta)\right]$. Then it is easily shown that

$$
0 \leq \frac{\partial H^{j+1}(K, \eta)}{\partial K} \leq\left[F_{1}(K, K, \eta)+F_{2}(K, K, \eta)\right]
$$

It is also seen from equation (3) that replacing $\mathrm{H}^{\mathrm{j}}(\mathrm{K}, \eta)$ by a function that is everywhere greater results in a larger solution for $\mathrm{x}$. In other words, this procedure generates a monotonically increasing sequence of laws of motion for the aggregate capital stock $\left(\mathrm{H}^{\mathrm{j}}(\mathrm{K}, \eta)\right\}^{\infty}{ }_{\mathrm{j}=0}$ defined on $[0, \overline{\mathrm{K}}] \times \Lambda$. Furthermore, this sequence is bounded from above by $\overline{\mathrm{K}}$ [see Assumption (ii)]. Let

$$
Q(K, \eta)=\lim _{j \rightarrow \infty} H^{j}(K, \eta)
$$

so that $Q(\cdot$,$) is the pointwise limit of the functions H^{j}(;$,$) which exists because \left\{H^{j}(K, \eta)\right\}^{\infty}{ }_{j=0}$ 
is a monotonically increasing bounded sequence. Since $\mathrm{H}^{\mathrm{j}}(0, \eta)=0$ for all $\mathrm{j}$, then $\mathrm{Q}(0, \eta)=0$ as well. Note from (4) that $\partial H^{j+1}(K, \eta) / \partial K$ is bounded from above by $F_{1}(K, K, \eta)+F_{2}(K, K, \eta)$, for all $j$, and hence the sequence $\left[\mathrm{H}^{\mathrm{j}+1}(\mathrm{~K}, \eta)\right\}^{\infty}{ }_{\mathrm{j}=0}$ is equicontinuous on $[\zeta, \overline{\mathrm{K}}]$ for all $\zeta \in(0, \overline{\mathrm{K}}]$. Therefore, the sequence $\left[\mathrm{H}^{\mathrm{j}+1}(\mathrm{~K}, \eta)\right\}_{\mathrm{j}=0}^{\infty}$ converges uniformly on each closed interval to $\mathrm{Q}(;)$, which must also be a function continuous in its first argument [by the Arzela-Ascoli theorem and theorem 7.13, Rudin (1976)]. ${ }^{3}$ Furthermore, $Q(; \cdot)$ must also satisfy the restriction given by equation (4), with the derivative interpreted as a finite difference.

Is the function $Q(K, \eta)=\lim _{j \rightarrow \infty} H^{\mathrm{j}}(K, \eta)$ constructed above a good candidate for an equilibrium aggregate law of motion? To answer this question, suppose that $Q(K, \eta)<F(K, K, \eta)$ for $K \in(0, \bar{K}]$ and $\eta \in \Lambda$. By construction, the function $K^{\prime}=Q(K, \eta)$ satisfies equation (2). Furthermore, given this aggregate law of motion, there exists a unique decision-rule $k^{\prime}=q(k, K, \eta)$ which solves the Euler equation (1). Clearly, when $k=K$, it transpires that $q(K, K, \eta)=Q(K, \eta)$ solves (1) since $Q(K, \eta)$ satisfied (2). Thus, for the case when $Q(K, \eta)<F(K, K, \eta)$ for all $K \neq 0$ an equilibrium has been found. To complete the existence proof it will be shown next that $Q(K, \eta)$ $=\lim _{\mathrm{j} \rightarrow \infty} \mathrm{H}^{\mathrm{j}}(\mathrm{K}, \eta) \neq \mathrm{F}(\mathrm{K}, \mathrm{K}, \eta)$ for $\mathrm{K} \in(0, \overline{\mathrm{K}}]$ and $\eta \in \Lambda$.

Remark: Observe that for any finite $T$, the sequence $\left\{H^{j}(K, \eta)\right\}^{T}=0$ gives the unique set of $T+1$ laws of motion that obtain from a T-period version of the economy under study.

\section{B. Nondegenerateness of the Aggregate Law of Motion}

It is important to ensure that the aggregate laws of motion do not converge to a degenerate case where investment equals total output. For instance, consider the possibility that $Q(K, \eta)=$ $F(K, K, \eta)$ for all $K$ and $\eta$. While this solution for the aggregate law of motion will trivially satisfy (2), it cannot constitute an equilibrium for the economy under study since (P1) demands an interior solution for the representative agent's decision-rule.

To rule out such degenerate cases, consider the sequence of value functions $\left[V^{n}\right]_{n=0}^{\infty}$ generated from the mapping

$$
V^{n}(k, K, \eta)=\max _{k}\left\{U\left(F(k, K, \eta)-k^{\prime}\right)+\beta \int V^{n-1}\left(k^{\prime}, K^{\prime}, \eta\right) d G\left(\eta^{\prime} \mid \eta\right)\right\},
$$


where $K^{\prime}=Q(K, \eta)$. Without loss of generality, assume that $V^{0}=0$. Well-known arguments can be employed to show that this mapping defines an operator $\mathrm{T}$, such that $\mathrm{V}^{\mathrm{n}}=\mathrm{TV}^{\mathrm{n}-1}$, that has as its unique fixed point the function $\mathrm{V}$ characterized by (P1). The following well-known fact will be stated for future use:

Fact 1: $\quad$ Since $V^{n} \rightarrow V$ uniformly, for all $\varepsilon>0$, there exists an $N$ such that for $n \geq N$

$$
\left|V^{n}(k, K, \eta)-V(k, K, \eta)\right| \leq \varepsilon / 3
$$

for all $k, K \in[0, \bar{K}], \eta \in \Lambda^{4}$

Now let $\left[Q^{i}\right]_{j=0}^{n}$ be a sequence of continuous functions converging uniformly to the function $Q$ on $[0, \bar{K}] \times \Lambda$. Next, consider the sequence of value functions $\left[\hat{V}^{n}\right\}_{n=0}^{\infty}$ generated from the mapping

$$
V^{n}(k, K, \eta)=\max _{k}\left\{U\left(F(k, K, \eta)-k^{\prime}\right)+\beta \int \hat{V}^{n-1}\left(k^{\prime}, K^{\prime}, \eta^{\prime}\right) d G\left(\eta^{\prime} \mid \eta\right)\right\}
$$

with $K^{\prime}=Q^{n}(K, \eta)$. Set $\hat{V}^{0} \equiv 0$. Let the optimal decision-rule associated with (P3) be denoted by $k^{\prime}=q^{n}(k, K, \eta)$. The key step in the subsequent analysis is to show that $\lim _{n \rightarrow \infty} \hat{V}^{n}=V$, where $V$ is the fixed point to equation (P1).

To this end, define the continuous function $\pi^{\mathrm{q}}:[0, \overline{\mathrm{K}}] \times \Lambda^{\mathrm{q}} \rightarrow \Re_{+}$recursively by

$$
\pi^{q}\left(K_{0}, \eta_{0}, \ldots \eta_{q-1}\right)=Q\left(\pi^{q-1}\left(K_{0}, \eta_{0}, \ldots \eta_{q-2}\right), \eta_{q-1}\right)
$$

where $\pi^{0} \equiv K_{0}$. The function $\pi^{q}$ gives the period-q aggregate capital stock assuming that the initial period-zero capital stock is $K_{0}$, the history of shocks $\left[\eta_{t}\right]^{q-1}{ }_{t=0}$ transpires, and the law of motion $Q$ is followed.

Likewise, define the continuous function $\AA^{m, q}:[0, \bar{K}] \times \Lambda^{q} \rightarrow \Re_{+}$, for $m \geq \max \{q, 1\}$, recursively by

$$
\hbar^{m, q}\left(K_{0}, \eta_{0}, \ldots \eta_{q-1}\right)=Q^{m-q}\left(\pi^{m, q-1}\left(K_{0}, \eta_{0}, \ldots \eta_{q-2}\right), \eta_{q-1}\right)
$$


with $\AA^{m, 0} \equiv K_{0}$. The function $\hat{\pi}^{m, q}$ gives the period-q aggregate capital stock for the economy with an m-period horizon, assuming that the law of motion $\mathrm{Q}^{\mathrm{m}-\mathrm{t}}$ is followed in period $t$ (for $t \leq \mathrm{q}-1$ ) and contingent upon the initial period-zero capital having the value $K_{0}$, and the history of shocks $\left\{\eta_{t}\right\}^{q-1}=0$ being realized. Note that since $Q^{j} \rightarrow Q$ uniformly, $\lim _{m \rightarrow \infty} \hat{\pi}^{m, q}=\pi^{q}$ for all $q>0$. This is easily seen to imply Fact 2 , which will be used in the subsequent analysis.

Fact 2: $\quad$ Since $U(\cdot)$ and $F(\cdot)$ are continuous functions, and the sequence $\left\{Q^{j}\right\}$ converges uniformly to $Q$, for all $q$ and $\varepsilon>0$, there exists an $M$ such that for $m \geq M \geq \max (q, 1)$,

$$
\left|U\left(F\left(k, \pi^{m, t}(\cdot), \eta_{t}\right)-k\right)-U\left(F\left(k, \pi^{t}(\cdot), \eta_{t}\right)-k\right)\right| \leq \frac{(1-\beta) \varepsilon}{3},
$$

for $\mathrm{k}, \mathrm{k}^{\prime}, \mathrm{K}_{0} \in[0, \overline{\mathrm{K}}],\left[\eta_{0}, \eta_{1}, \ldots \eta_{\mathrm{t}}\right] \in \Lambda^{\mathrm{t}+1}$, and $0 \leq \mathrm{t} \leq \mathrm{q}$.

Finally, Fact 3 (which is trivial) is noted.

Fact 3: $\quad$ Let $B \equiv \max _{\eta \varepsilon \Lambda}[U(F(\bar{K}, \bar{K}, \eta))]$. For each $\varepsilon>0$, there exists a $P$ such that for all $\mathrm{p} \geq \mathrm{P}$,

$$
\left(\frac{\beta^{p}}{1-\beta}\right) B<(\varepsilon / 3)
$$

Lemma: $\quad \operatorname{Lim}_{n \rightarrow \infty} \hat{V}^{n}=V$.

Proof: $\quad$ Pick $\varepsilon>0$ and choose $N$ and $P$ such that Facts 1 and 3 hold. Next, let $q=\max [N, P]$ and choose $M \geq \max (q, 1)$ large enough so that Fact 2 holds as well. Now note that

$$
\hat{V}^{m}\left(k_{0}, K_{0}, \eta_{0}\right)=\max _{\left\{k_{t+1}\right\}_{t=0}^{m}} E\left(\sum_{t=0}^{m} \beta^{t} U\left(F\left(k_{t}, \hat{\pi}^{m, t}, \eta_{t}\right)-k_{t+1}\right)\right),
$$

where the expectation operator $\mathrm{E}(\cdot)$ reflects the integration with respect to the finite probability distribution of $\left\{\eta_{j}\right\}_{j=1}^{m}$. First, set $m \geq M$. By Fact 3 it follows that 


$$
\left|V\left(k_{0}, K_{0}, \eta_{0}\right)-\hat{V}^{m}\left(k_{0}, K_{0}, \eta_{0}\right)\right| \leq\left|V\left(k_{0}, K_{0}, \eta_{0}\right)-\max _{\left\{k_{t+1}\right]_{t=0}^{q}} E\left(\sum_{t=0}^{q} \beta^{t} U\left(F\left(k_{t}, \hat{\pi}^{m, t}, \eta_{t}\right)-k_{t+1}\right)\right)\right|+\frac{\varepsilon}{3},
$$

since $q \geq P$. Second, Fact 2 then implies

$$
\begin{aligned}
& \left|V\left(k_{0}, K_{0}, \eta_{0}\right)-\hat{V}^{m}\left(k_{0}, K_{0}, \eta_{0}\right)\right| \leq\left|V\left(k_{0}, K_{0}, \eta_{0}\right)-\max _{\left\{k_{t+1}\right\}_{t=0}^{q}} E\left(\sum_{t=0}^{q} \beta^{t} U\left(F\left(k_{t}, \pi^{t}, \eta_{t}\right)-k_{t+1}\right)\right)\right|+\left(\frac{2 \varepsilon}{3}\right) \\
& =\left|V\left(k_{0}, K_{0}, \eta_{0}\right)-V^{q}\left(k_{0}, K_{0}, \eta_{0}\right)\right|+\left(\frac{2 \varepsilon}{3}\right) .
\end{aligned}
$$

Third, since $q>N$, it immediately transpires from Fact 1 that

$$
\left|V\left(k_{0}, K_{0}, \eta_{0}\right)-\hat{V}^{m}\left(k_{0}, K_{0}, \eta_{0}\right)\right| \leq \varepsilon .
$$

It will now be established that $Q(K, \eta)<F(K, K, \eta)$ for $K \in(0, \bar{K}]$ and $\eta \in \Lambda$. Note that the sequence of functions $\left[\mathrm{H}^{\mathrm{n}}\right\}_{\mathrm{n}=0}^{\infty}$ constructed in the previous subsection satisfies the properties assumed for the sequence $\left\{Q^{n}\right\}^{\infty}{ }_{n=0}$ that underlies the mapping (P3).. Therefore let $\mathrm{Q}^{\mathrm{n}}=\mathrm{H}^{\mathrm{n}}$ for all n. Then by the construction of mapping (P3), $q^{n}(K, K, \eta)=H^{n}(K, \eta)$ for $K \in[0, \bar{K}]$ and $\eta \in \Lambda$. Now, consider the sequence of functions $\left\{W^{n}\right\}^{\infty}{ }_{n=0}$ where $W^{n}\left(k^{\prime}, K, \eta, \eta^{\prime}\right) \equiv \hat{V}^{n}\left(k^{\prime}, Q^{n-1}(K, \eta), \eta^{\prime}\right)$. Clearly, this sequence converges to the function $W\left(k^{\prime}, K, \eta, \eta^{\prime}\right)=V\left(k^{\prime}, Q(K, \eta), \eta^{\prime}\right)$ by the lemma presented above. Since the functions $\mathrm{W}$ and $\mathrm{W}^{\mathrm{n}}$, for all $\mathrm{n}$, are each strictly concave in their first argument it follows that $\mathrm{q}^{\mathrm{n}}(\mathrm{k}, \mathrm{K}, \eta) \rightarrow \mathrm{q}(\mathrm{k}, \mathrm{K}, \eta)$, pointwise. Consequently, $\mathrm{Q}(\mathrm{K}, \eta)<\mathrm{F}(\mathrm{K}, \eta)$ for $\mathrm{K}$ $\neq 0$, since problem $(\mathrm{P} 1)$ has an interior solution for its decision-rule $\mathrm{q}(\mathrm{k}, \mathrm{K}, \eta)$.

\section{Uniqueness of the Aggregate Law of Motion}

It will now be established that the nontrivial equilibrium for the economy under study is unique. ${ }^{5}$ To see this, suppose that some other law of motion $K^{\prime}=M(K, \eta)$ and decision-rule $k^{\prime}$ $=\mathrm{m}(\mathrm{k}, \mathrm{K}, \eta)$ satisfies the definition for an equilibrium presented above. Obviously, it must then be the case that $Q(K, \eta) \neq M(K, \eta)$ for some $(K, \eta)$; pick a $(K, \eta)$ for which this is true. For this $(K, \eta)$ pair, 


$$
U^{\prime}\left(F(K, K, \eta)-\tilde{K}^{\prime}\right)=\beta \int U^{\prime}\left(F\left(\tilde{K}^{\prime}, \tilde{K}^{\prime}, \eta^{\prime}\right)-\tilde{K}^{\prime \prime}\right) F_{1}\left(\tilde{K}^{\prime}, \bar{K}^{\prime}, \eta^{\prime}\right) d G\left(\eta^{\prime} \mid \eta\right)
$$

where $\tilde{K}^{\prime}=M(K, \eta)$ and $\tilde{K}^{\prime \prime}=M\left(\tilde{K}^{\prime}, \eta\right)$. Now, suppose that $Q(K, \eta)<M(K, \eta)$. It is easy to see that the left-hand side of (6) must be greater than that of equation (2). Thus, so must be the right-hand side. Assumption (iii) can be used to show that this implies

$$
\frac{\partial M(K, \eta)}{\partial K}>\left[F_{1}(K, K, \eta)+F_{2}(K, K, \eta)\right]
$$

But this contradicts (4), and thus the situation being entertained cannot transpire. The case where $\mathrm{Q}(\mathrm{K}, \eta)>\mathrm{M}(\mathrm{K}, \eta)$ leads to a similar contradiction.

\section{DISCUSSION}

The form for the production technology, $y=F(k, K, \eta)$, is a general formulation that could embody many environments. Three examples may help to illustrate this point. Additionally, one counterexample will be shown in which the proposed assumptions are not satisfied, and there exists multiple equilibria.

Example 1: Distortional Taxation. Let the representative agent's production function be described by $y=Y(k, \eta)+(1-\delta) k$, where $Y$ has the standard production function properties. Note that under this formulation capital depreciates at the rate $\delta$. Now suppose that there is a government in the economy which taxes capital income at the rate $\theta$ and provides a capital consumption allowance of $\mu$. The resulting tax revenue is rebated back to the agent via a lump-sum transfer payment. This environment can be handled in the above framework by adopting the following specification for $F(k, K, \eta)$ :

$$
F(k, K, \eta)=(1-\theta) Y(k, \eta)+[1-\delta(1-\mu)] k+\theta Y(K, \eta)-\mu \delta K .
$$

It is straightforward to check that Assumptions (i), (ii) and (iii) are satisfied. Additionally, this framework can also accommodate a progressive income tax structure. This can be done by letting the capital income tax rate depend on the aggregate capital stock $\theta(\mathrm{K})$. It is easily seen that as long as the tax is progressive, so that $\theta^{\prime}(\mathrm{K})>0$, the required assumptions are satisfied. 
Furthermore, it is easy to incorporate a variable supply of labor into the analysis. Now, let the representative agent's preferences be described by $U(c-G(\ell))$, where $G(\cdot)$ is an increasing convex function. ${ }^{6}$ The technology is specified as $y=Y(k, \ell, \eta)$, where $\ell$ represents his labor input. Also, suppose that the government taxes labor income at the rate $\lambda$. Define the function $L(k, \eta)$ by

$$
L(k, \eta)=\underset{x}{\operatorname{argmax}}[(1-\theta) Y(k, x, \eta)-[(1-\theta) /(1-\lambda)] G(x)\}
$$

To recast the environment into the framework developed, let $F(k, K, \eta)$ be given by

$$
\begin{gathered}
F(k, K, \eta)=(1-\theta) Y(k, L(k, \eta), \eta)+[1-\delta(1-\mu)] k-[(1-\theta) /(1-\lambda)] G(L(k, \eta)) \\
+\theta Y(K, L(K, \eta), \eta)-\mu \delta K+[(\lambda-\theta) /(1-\lambda)] G(L(K, \eta)) .
\end{gathered}
$$

While now a little more difficult to verify, $F(k, K, \eta)$ satisfies Assumptions (i), (ii) and (iii). The representative agent's dynamic programming problem can again be characterized as (P1), given this formulation for $F(k, K, \eta)$.

Example 2: Endogenous Growth with Externalities. Consider a simple version of Romer's (1986) well-known economic growth model. First, let representative agent's preferences be described by $\sum_{t=0}^{\infty} \beta^{t} \ln c_{t}$. Second, suppose the production technology is given by $\mathrm{y}=\mathrm{Y}(\mathrm{k}, \mathrm{K}, \eta)$,

where $\mathrm{Y}$ is linearly homogeneous in its first two arguments with the standard properties holding otherwise. With this combination of tastes and technology it is possible for the economy to grow without bound. The model, however, has a stationary representation which can be handled in the framework developed above. To see this, define the variables $\hat{c}, \hat{k}^{\prime}$, and $\hat{K}^{\prime}$ by $\hat{c}=c / K, \hat{k}^{\prime}=k^{\prime} / K$, and $\hat{K}^{\prime}=K^{\prime} / K$. Next, let $F(\hat{k}, \hat{K}, \eta)$ be represented by $F(\hat{k}, \hat{K}, \eta)=Y(\hat{k} / \hat{K}, 1, \eta)$. Observe that $F$ satisfies Assumptions (i), (ii) and (iii), with $F_{1}+F_{2}=0$ when $\hat{k}=\hat{K}$.

Now, it is easy to deduce that the representative agent's dynamic programming problem (P1) can be rewritten for the current example in transformed form as 


$$
V(\hat{k}, \hat{K}, \eta)=\max _{\hat{K^{\prime}}}\left\{\ln \left[F(\hat{k}, \hat{K}, \eta)-\hat{k}^{\prime}\right]+\beta \int V\left(\hat{k}^{\prime}, \hat{K}^{\prime}, \eta^{\prime}\right) d G\left(\eta^{\prime} \mid \eta\right)\right\}
$$

where $\hat{K}^{\prime}=Q(\hat{K}, \eta)$. Since $\mathrm{F}_{1}+\mathrm{F}_{2}=0$, when $\hat{\mathrm{k}}=\hat{\mathrm{K}}$ it is immediate from (4) that the equilibrium law of motion can be expressed more simply as $\hat{K}=Q(\eta)$. Clearly then, the solution for the original model is homogeneous of degree one in the aggregate capital stock. Also, it is easy to check by solving (P4) that

$$
Q(\eta)=\beta\left(\int\left[F_{1}\left(1,1, \eta^{\prime}\right) / F\left(1,1, \eta^{\prime}\right)\right] d G\left(\eta^{\prime} \mid \eta\right)\right) F(1,1, \eta) .
$$

The economy can expect long-run growth if $F$ and $G$ are such that $E\left(\hat{\mathrm{K}}^{\prime}\right)=\int Q(\eta) d \Gamma(\eta)>1$, where $\Gamma$ is the fixed point to $\Gamma\left(\eta^{\prime}\right)=\int G\left(\eta^{\prime} \mid \eta\right) \mathrm{d} \Gamma(\eta)$. Observe that when the technology shock takes Hicks neutral form, growth can be expected to occur when $\beta E[\eta] F_{1}(1,1)>1$, i.e., when the expected real interest exceeds the discount factor. ${ }^{7}$ Finally, suppose that $F_{1}(1,1, \eta)$ is increasing in $\eta$ and $F_{1}(1,1, \eta) / F(1,1, \eta)$ is nondecreasing. Also, let $G$ exhibit positive serial correlation in the

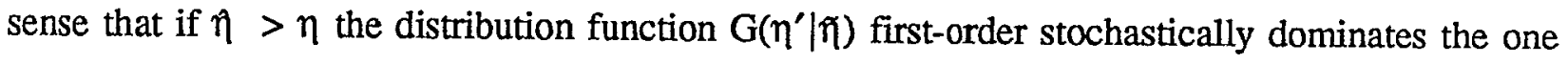
given by $G\left(\eta^{\prime} \mid \eta\right)$. Then, under these assumptions, $Q(\eta)$ is increasing in $\eta^{8}$ [For the Hicks neutral case $Q(\eta)$ is unambiguously increasing in $\eta$.

Example 3: Monopolistic Competition. A simple stochastic infinite horizon version of Kiyotaki's (1988) model of monopolistic competition also fits into the framework developed. Imagine an economy inhabited by a continuum of agents distributed uniformly over the unit interval. Agents are identical except that each owns a firm producing a differentiated product. The generic individual's preferences are described by $\sum_{t=0}^{\infty} \beta^{t} \ell n\left[\int_{0}^{1} c_{t}(\theta)^{\rho} d \theta\right]^{\frac{1}{\rho}}$, for $0<\rho<1$, where $c_{t}(\theta)$ represents his period-t consumption of the good produced by agent $\theta$. The representative agent's firm produces output according to the linear production technology $y=\eta \mathrm{k}$, where $\eta$ is an aggregate technology shock. An individual's capital accumulation is governed by the technology $\mathrm{k}^{\prime}=$ $\left[\int_{0}^{1} \mathrm{i}(\theta)^{\rho} \mathrm{d} \theta\right]^{1 / \rho}$, where $\mathrm{i}(\theta)$ is the amount of goods purchased from agent $\theta$ for investment purposes. Finally, the agent's budget constraint for any given period reads $\int_{0}^{1} \mathrm{p}(\theta)[\mathrm{c}(\theta)+\mathrm{i}(\theta)] \mathrm{d} \theta=\mathrm{p} \eta \mathrm{k}$, where 
$\mathrm{p}(\theta)$ is the price for good $\theta$.

Now, by carrying out the implied within-period maximization, it is easy to deduce that $c(\theta)=[\mathrm{p}(\theta) / \mathrm{P}]^{1 /(\rho-1)}\left[\int_{0}^{1} \mathrm{p}(\xi) c(\xi) d \xi / P\right]$ and $\mathrm{i}(\theta)=[\mathrm{p}(\theta) / \mathrm{P}]^{1 /(p-1)}\left[\int_{0}^{1} \mathrm{p}(\xi) \mathrm{i}(\xi) \mathrm{d} \xi / \mathrm{P}\right]$, with the aggregate price level $P$ being given by $\left[\int_{0}^{1} p(\xi)^{\rho /(\rho-1)} d \xi\right]^{(\rho-1) / \rho}$. Next, define $c$ and $k^{\prime}$ by $c=\left[\int_{0}^{1} c(\theta)^{\rho} d \theta\right]^{1 / \rho}$ and $k^{\prime}=\left[\int_{0}^{1} \mathrm{i}(\theta)^{\rho} \mathrm{d} \theta\right]^{1 / \rho}$. The agent's preferences can now be represented more simply by $\sum_{t=0}^{\infty} \beta^{t} \ell n c_{t}$. Also, using the above formulae it is apparent that $c=\int_{0}^{1} \mathrm{p}(\theta) \mathrm{c}(\theta) \mathrm{d} \theta / \mathrm{P}$ and $\mathrm{k}^{\prime}=$ $\int_{0}^{1} \mathrm{p}(\theta) \mathrm{i}(\theta) \mathrm{d} \theta / \mathrm{P}$. This allows the individual's budget constraint to be given the more compact representation $c+k^{\prime}=(p / P) \eta k$. Observe that the demand, $y$, for the agent's firm's product is described by $y=(p / P)^{1 /(p-1)} Y$, where aggregate demand $Y$ is defined by $Y=\int p(\theta)[C(\theta)+I(\theta)] d \theta / P$ and $C(\theta)$ and $I(\theta)$ represent the aggregate amounts of good $\theta$ that are demanded for consumption and investment purposes. Thus, $\mathrm{p} / \mathrm{P}=(\eta \mathrm{k} / \mathrm{Y})^{\rho-1}$, as $\mathrm{y}=\eta \mathrm{k}$. Since in equilibrium each industry behaves the same, it must transpire that $\mathrm{Y}=\eta \mathrm{K}$, where $\mathrm{K}$ is the average or aggregate level of the capital stock. Therefore the representative agent's budget constraint can be reformulated as $c+k^{\prime}$ $=\eta \mathrm{k}^{\rho_{\mathrm{K}}} \mathrm{K}^{1-\rho}$. The agent's intertemporal optimization problem amounts to choosing $\mathrm{c}$ and $\mathrm{k}^{\prime}$ in each period so as to maximize expected lifetime utility. The problem now has the form of Example 2. Observe that the economy can grow without bound.

Example 4: A Counterexample. It may be enlightening to see that if the proposed assumptions are not in place, then there exists the possibility of multiple equilibria occurring. Therefore, let the technology be of the following form:

$$
F(k, K, \eta)=30 \eta k^{\alpha} H(K),
$$

where $\mathrm{H}$ is given the specification below.

$$
H(K)= \begin{cases}1 & \text { for } k \in[0,10] \\ 2+\cos [-\pi+k(\pi / 5)] & \text { for } k \in[10,15] \\ 3 & \text { for } k \geq 15\end{cases}
$$

Note that $\mathrm{F}(\cdot)$ is differentiable and increasing everywhere, satisfies Assumption (ii), but does not satisfy Assumption (iii). This is because the technology exhibits decreasing, increasing, and then decreasing social returns in the individual capital stock over successive regions. Let $\beta=2 / 3, \alpha=$ .20 , and $\eta_{t}=1$ for all $t$. Figure 1 portrays the resulting production technology along the 
equilibrium path, while Figure 2 shows the private marginal product of capital along the equilibrium path. ${ }^{9}$ This (non-stochastic) economy has three equilibria. Two of these are stable, one with $\mathrm{K}$ $\approx 5.69$, and one with $\mathrm{K} \approx 22.34$, and one is unstable with $\mathrm{K} \approx 12.28$. Of course, if uncertainty is introduced through $\eta_{\mathrm{t}}$ this could result in two stationary distributions for the capital stock. 


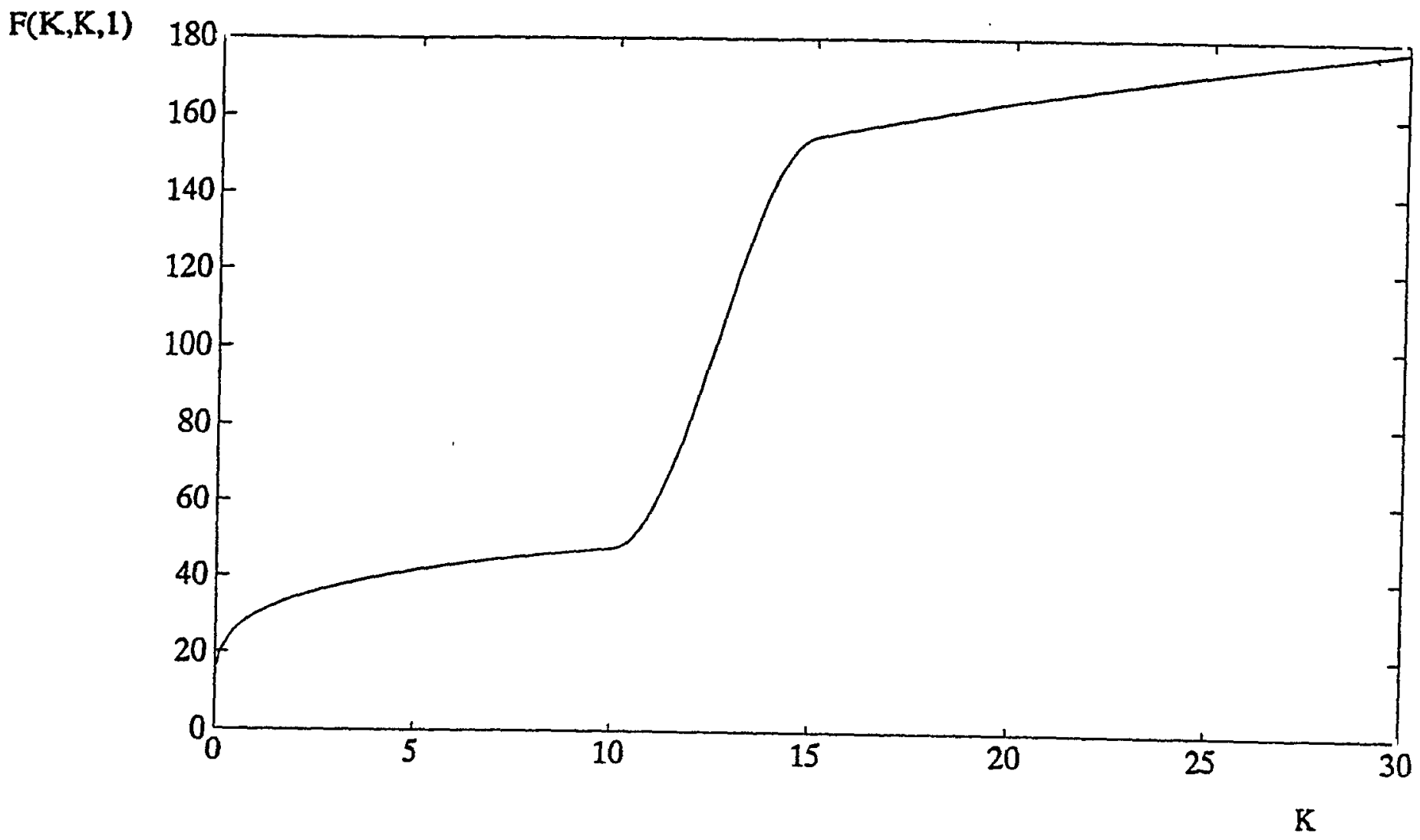

Figure 1: Production Function 


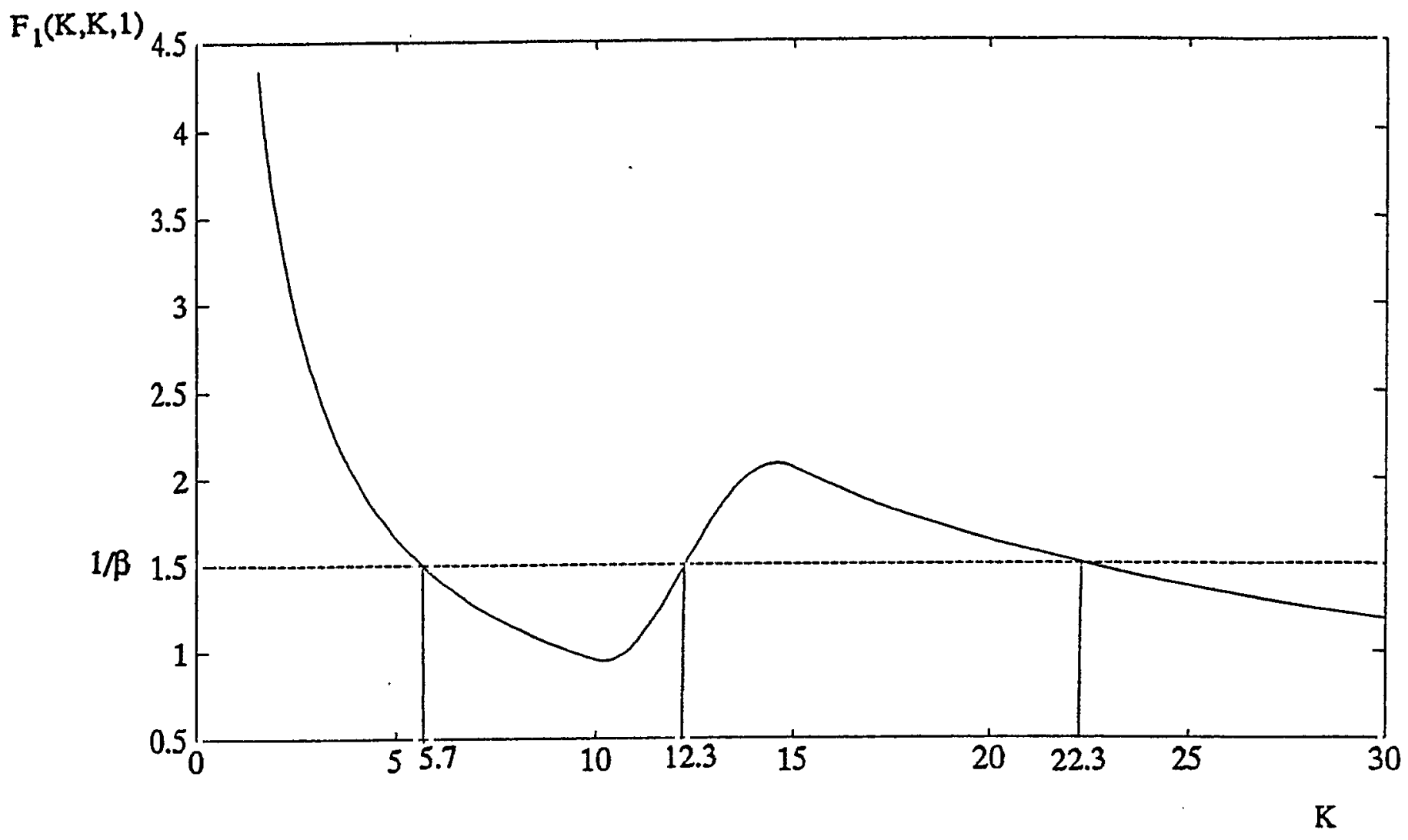

Figure 2: Private Marginal Product of Capital 


\section{FOOTNOTES}

1. In particular, Assumption 5 in Coleman (1991) assumes that there is not "too much" uncertainty about the production function disturbances. No such assumption is needed in the present analysis, as the degree of uncertainty is unrestricted.

2. This mapping forms the basis for numerical algorithms to solve nonlinear dynamic stochastic models that have been proposed by Baxter (1991), Coleman (1990), and Danthine and Donaldson (1990).

3. Specifically, the Arzela-Ascoli theorem implies that the limiting function is continuous in its first argument, while the theorem in Rudin (1976) establishes that the convergence is uniform since the sequence is monotone.

4. Here the norm employed is $|\mathrm{V}|=\sup _{\mathrm{x}}\left|\mathrm{V}\left(\mathrm{x}_{1}, \mathrm{x}_{2}, \mathrm{x}_{3}\right)\right|$, where $\mathrm{x}=\left(\mathrm{x}_{1}, \mathrm{x}_{2}, \mathrm{x}_{3}\right) \in$ $[0, \bar{K}] \times[0, \bar{K}] \times \Lambda$. Similarly, the norm for the convergence of the functions $\left\{Q^{j}\right\}_{j=0}^{\infty}$ is also the sup norm.

5. Depending on the form of $F(k, K, \eta)$, there may also exist trivial equilibria where $Q(K, \eta)=0$. These equilibria can be ruled out by adding the assumption that $F(k, 0, \eta)>0$ for $k>0$. This assumption is fairly weak, and is satisfied in Coleman's (1991) analysis for instance. Now the individual will always choose to invest something, notwithstanding the fact that nobody else is.

6. This form of utility function has been used in Greenwood, Hercowitz and Huffman (1988) and Hercowitz and Sampson (1991). These analyses suggest that for the study of business cycles the restrictions imposed by this sort of utility may not be that severe.

7. For the Hicks' neutral case let $F(k, K, \eta)$ be represented by $\eta F(k, K)$.

8. For more information on the concept of stochastic dominance, see Hadar and Russell (1971).

9. Note that $\partial \mathrm{F}_{1}(\mathrm{~K}, \mathrm{~K}, \eta) / \partial \mathrm{K}=\mathrm{F}_{11}(\mathrm{~K}, \mathrm{~K}, \eta)+\mathrm{F}_{21}(\mathrm{~K}, \mathrm{~K}, \eta)$. 


\section{REFERENCES}

Baxter, Marianne, "Approximating Suboptimal Dynamic Equilibria: An Euler Equation Approach," Journal of Monetary Economics, 28 (October 1991): 173-200.

Coleman, Wilbur John II, "Equilibrium in a Production Economy with an Income Tax" Econometrica, 59 (July 1991): 1091-1104.

Coleman, Wilbur John II, "Solving the Stochastic Growth Model by Policy-Function Iteration," Journal of Business and Economic Statistics, 8 (January 1990): 27-29.

Danthine, Jean-Pierre and Donaldson, John B., "Efficiency Wages and the Business Cycle Puzzle," European Economic Review, 34 (November 1990): 1275-1301.

Greenwood, Jeremy, Zvi Hercowitz and Gregory W. Huffman, "Investment, Capacity Utilization, and the Real Business Cycle," American Economic Review, 78 (June 1988): 402-417.

Greenwood, Jeremy and Gregory W. Huffman, "Tax and Stabilization Policies in a Dynamic Economy," unpublished paper, London: University of Western Ontario, 1991. Forthcoming in John B. Shoven and John Whalley, eds., Applied General Equilibrium Modelling, Cambridge University Press.

Hadar, Josef and Russell, William R., "Stochastic Dominance and Diversification," Journal of Economic Theory, 3 (September 1971): 288-305.

Hercowitz, Zvi and Michael Sampson, "Output Growth, the Real Wage, and Employment Fluctuations," American Economic Review, 81 (December 1991): 1215-1237.

Kiyotaki, Nobuhiro, "Multiple Expectational Equilibria Under Monopolistic Competition," Quarterly Journal of Economics CIII (November 1988): 695-713.

Romer, Paul M., "Increasing Returns and Long-Run Growth," Journal of Political Economy, 94 (October 1986): 1002-1037.

Rudin, Walter, Principles of Mathematical Analysis, McGraw Hill, Inc., New York, (1976). Stokey, Nancy L., and Robert E. Lucas Jr., with Edward C. Prescott, Recursive Methods in Economic Dynamics, Harvard University Press, 1989. 